\title{
Edge imaging in intense beams
}

\author{
S. Bernal, B. Quinn, M. Reiser,* and P. G. O'Shea* \\ Institute for Research in Electronics and Applied Physics, University of Maryland, College Park, Maryland 20742
}

(Received 5 December 2001; revised manuscript received 16 May 2002; published 19 June 2002)

\begin{abstract}
The appearance of rings of charge observed near the edge of beams from high-perveance guns is described with a simple ray tracing technique inspired by the particle-core model. We illustrate the technique, which has no analog in light optics, with examples from experiments employing solenoid focusing of an electron beam. The rings of charge result from the combined effects of external focusing and space-charge forces acting on paraxial fringe particles with relatively large initial transverse velocities. The model is independent of the physical mechanisms responsible for the fringe particles. Furthermore, the focal length for edge imaging in a uniform focusing channel is derived using a linearized trajectory equation for the motion of fringe particles. Counterintuitively, the focal length decreases as the beam current increases.
\end{abstract}

DOI: 10.1103/PhysRevSTAB.5.064202

PACS numbers: 41.85.Ew, 29.27.Bd

\section{INTRODUCTION}

The optics of charged-particle beams has been the subject of extensive study for many years [1]. In these studies, a close analogy with ray optics has been used, which is justified by the relatively weak beam currents or the high energies of most relevant applications. Charged-particle optics that include the effects from self-fields, on the other hand, has been the subject of more recent investigations [2]. Two theoretical advances have played a major role in understanding the effects of self-fields, thus facilitating the design of low-energy, high-current beam transport systems: the Kapchinskij-Vladimirskij (or KV) particle distribution [3] and the concept of equivalent beams [4]. The KV distribution, characterized by uniform particle densities in both space and velocity, is widely used to model intense beams. The concept of equivalent beams, in turn, states that a KV distribution can be used to approximate any distribution if the beam boundaries and emittances of the two distributions are the same in a root-mean-square (rms) sense. On the computational front, the 2D particlecore model (PCM) [5,6] employs a KV distribution for the beam core and "external" test particles for studies of beam stability and halo formation in focusing lattices, under various conditions of beam matching and current.

We have used a construction inspired by the PCM to model the emergence of rings of charge in experiments and simulations $[7,8]$. These rings are to be distinguished from those resulting from spherical aberration [9], or the spatial structures reported in connection to wave breaking in phase space [10]. We present in this paper new experimental results and theoretical insights to support the existence of "edge imaging." The phenomenon involves the focusing of fringe particles at a distance significantly

\footnotetext{
*Also at Department of Electrical and Computer Engineering, University of Maryland.
}

different than the corresponding distance for particles originating near the beam core. Although edge imaging can be reproduced in particle-in-cell computer simulations starting with a semi-Gaussian (SG) particle distribution $[7,8]$, we focus our attention here on experimental results and on a simple model that illustrates the role of space charge and the scaling of the focal distance. In the limit of a laminar beam, the focal length for edge imaging in a uniform focusing channel is equal to one-half the plasma wavelength, $\lambda_{P} / 2$ (Sec. III), while edge imaging becomes indistinguishable from the better known "core imaging" in the limit of zero space charge. In this case, the focal distance is equal to one-half the undepressed betatron wavelength, $\lambda_{0} / 2$.

We present in the next section results from experiments with a short solenoid, along with corresponding edgeimaging constructions inspired by the PCM. Section III introduces edge imaging in the context of the smooth approximation, concluding with a simple expression for the focal distance. The last section is devoted to conclusions.

\section{EDGE IMAGING WITH A SHORT SOLENOID}

The main experiment setup is illustrated in Fig. 1. A $5 \mathrm{keV}$ electron beam with a pulse length of about $100 \mathrm{~ns}$ is focused by a short solenoid placed $16.1 \mathrm{~cm}$ from the beam waist, or about $19 \mathrm{~cm}$ from the thermionic, planar cathode ( $8 \mathrm{~mm}$ diameter) of a Pierce-type gridded gun. A rotatable plate with a number of apertures is employed to collimate the beam near the beam waist, close to the gun exit plane. We used four apertures with radii equal to 8 (full beam), $2.85,1.5$, and $0.875 \mathrm{~mm}$. Table I summarizes the beam parameters. The effective (i.e., $2 \mathrm{rms}$ ) beam radius of the full beam at the aperture plane is $3.2 \mathrm{~mm}$; it is obtained from a study of fluorescent screen pictures of the freely expanding beam. A Pearson transformer is also employed for beam current measurements.

The beam parameters and solenoid's strength are chosen in a compromise to reduce possible effects from the 


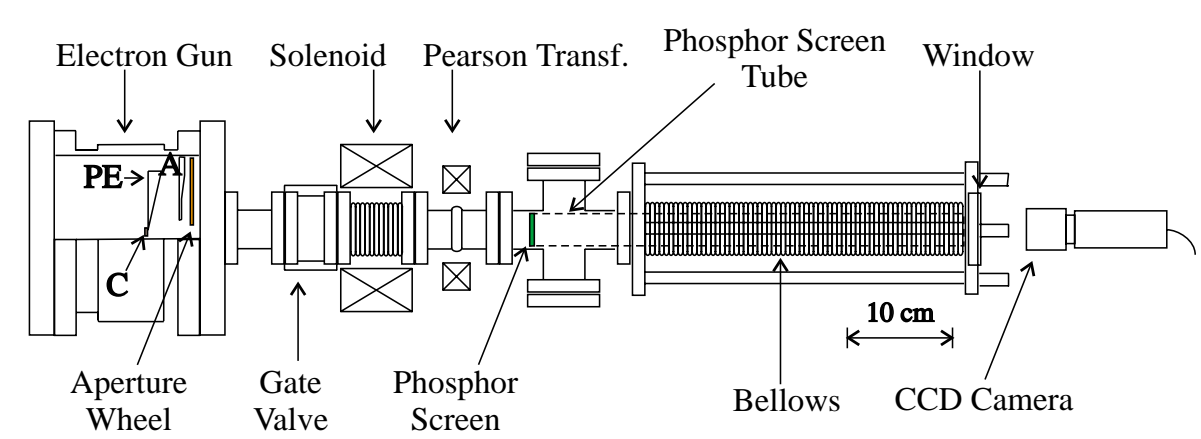

FIG. 1. Solenoid experiment setup. The cutout on the electron gun shows the cathode (C), Pierce focusing electrode (PE), and anode (A). The gridded electron gun has a mesh (not shown) in front of the cathode. In the setup for the convergent-type gun, a gasket with a 1/4-in. (diam) hole is used to collimate the beam at the downstream side of the gate valve.

solenoid's nonlinearities while obtaining a suitable beam size and good dynamical range in the fluorescent screen diagnostic. The latter consists of a $2.5 \mathrm{~cm}$ (diameter) screen with P-43 phosphor coating. The screen can be moved from $5 \mathrm{~cm}$ to almost $30 \mathrm{~cm}$ out from the plane of the aperture plate. An intensified charge-coupled device (CCD) camera, computer video capture, and associated software for image processing are also used. Issues related to linearity and calibration are discussed in Ref. [11].

Figures $2 a-2 d$ show the evolution of the beam over $30 \mathrm{~cm}$, starting at $6.4 \mathrm{~cm}$ (Figs. 2a-2c) downstream from the plane of the apertures (the closest distance made possible by mechanical constraints). The bottom parts of the figures display the normalized, "straight cut" horizontal beam profiles as well as the equivalent $\mathrm{KV}$ profiles. It is clear from Fig. 2a that the sharp-edged full beam yields the maximum hollowness in the vicinity of $z=29 \mathrm{~cm}$, before the beam waist. In contrast, Figs. $2 \mathrm{c}$ and $2 \mathrm{~d}$ show that hollowness is small or absent for the smallest beam currents. In Fig. 2d, the pictures appear equally intense because the optics were adjusted in each case to operate near saturation of the CCD sensor. The beam profiles in Fig. 2d, however, are normalized to the profile corresponding to the last picture on the right.

In another experiment, employing a convergent-type Pierce gun, a $4 \mathrm{keV}, 175 \mathrm{~mA}$ electron beam ( $5 \mu$ s pulse) is passed through an aperture $6.4 \mathrm{~mm}$ in diameter, placed
$12.4 \mathrm{~cm}$ from a thermionic concave cathode $(25.4 \mathrm{~mm}$ diameter). The aperture is roughly $1 / 3$ of the full beam size at that plane and results in an almost uniform, $17 \mathrm{~mA}$ beam entering the transport pipe. In this second experiment, the same short solenoid as in the first experiment is used, but placed $12.5 \mathrm{~cm}$ from the aperture. This time, and as seen in Fig. 3, the initially uniform profile of the collimated beam remains fairly uniform for a relatively long distance, becoming moderately hollow at about $z=43 \mathrm{~cm}$ from the aperture.

To further illustrate the effect of solenoid focusing, Figs. $4 \mathrm{a}$ and $4 \mathrm{~b}$ show the calculated envelope evolutions as well as the experimentally determined beam radii for experiments with the two electron guns. The differential equation for the beam envelope $R(z)$ is given by (see, for example, [2])

$$
R^{\prime \prime}(z)+k_{0}^{2}(z) R(z)-\frac{K}{R(z)}-\frac{\varepsilon^{2}}{R^{3}(z)}=0,
$$

where $K=2\left(I / I_{0}\right)\left(1 / \beta^{3} \gamma^{3}\right)$ is the generalized beam perveance, $I_{0}=17 \mathrm{kA}$, approximately, for electrons, and $\beta=v / c, \gamma=\left(1-\beta^{2}\right)^{-1 / 2}$ ( $c$ is the speed of light, and $v$ is the particle's velocity). The solenoid on-axis peak fields are $B_{01}=84.2 \mathrm{G}$ (gridded gun experiment), and $B_{02}=63.5 \mathrm{G}$ (convergent gun experiment), corresponding to peak focusing $k_{01}=17.6 \mathrm{~m}^{-1}$, and $k_{02}=14.9 \mathrm{~m}^{-1}$ for an effective solenoid's length equal to $4.5 \mathrm{~cm}$. The

TABLE I. Beam parameters in solenoid experiments.

\begin{tabular}{lcccc}
\hline \hline & $\begin{array}{c}\text { Beam radius, }{ }^{\mathrm{a}} R \\
(\mathrm{~mm})\end{array}$ & $\begin{array}{c}\text { Beam current } \\
(\mathrm{mA})\end{array}$ & $\begin{array}{c}\text { Emittance, }{ }^{\mathrm{b}} \boldsymbol{\epsilon} \epsilon \\
(\mu \mathrm{m})\end{array}$ & $K R^{2} / \varepsilon^{2 \mathrm{c}}$ \\
\hline Full beam $1^{\mathrm{d}}$ & 3.2 & $42.6 \pm 5 \%$ & $71 \pm 10 \%$ & 3.7 \\
Aperture 1 & 2.85 & 23.5 & 68 & 1.8 \\
Aperture 2 & 1.5 & 6.5 & 30 & 0.69 \\
Aperture 3 & 0.875 & 1.9 & 20 & 0.15 \\
Full beam 2 & 3.2 & 17.0 & 67 & 2.2 \\
\hline \hline
\end{tabular}

\footnotetext{
${ }^{\mathrm{a}}$ At aperture plane, $z=0$.

b4 rms, unnormalized.

${ }^{\mathrm{c}}$ At aperture plane, $z=0 ; K$ defined below Eq. (1).

${ }^{\mathrm{d}}$ Gridded gun.

${ }^{\mathrm{e} C}$ Convergent gun.
} 

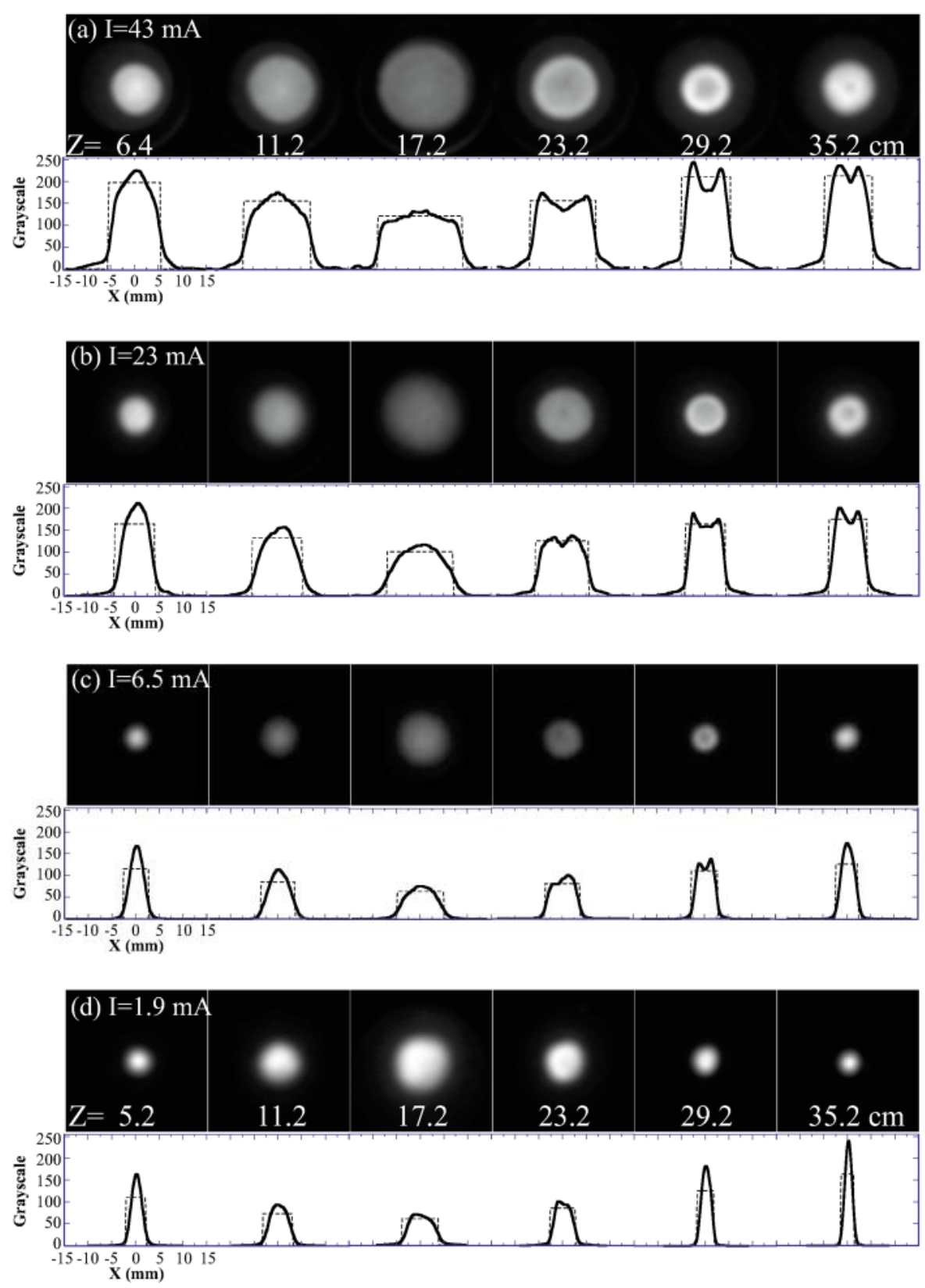

FIG. 2. (Color) Solenoid focusing of a $5 \mathrm{keV}$ electron beam for different currents (see Table I). The fluorescent screen pictures and normalized horizontal intensity profiles are shown for (a) full beam (43 mA), (b) $2.85 \mathrm{~mm}$ (radius) aperture, (c) $1.5 \mathrm{~mm}$ (radius) aperture, and (c) $0.875 \mathrm{~mm}$ (radius) aperture. The distance $z$ is measured from the aperture plate (see Fig. 1). The solenoid is located at $z=16.1 \mathrm{~cm}$ and has a peak on-axis field $B_{z}=84.2 \mathrm{G}$. The vertical scales on the beam profiles are in units of a gray scale where 0 is black and 256 is white. The dotted rectangular plots represent the equivalent $K V$ profiles.

solenoid focusing is implemented with a smooth profile that is a close fit to the actual square field (see Fig. 4).

The effect of space charge relative to thermal effects can be estimated as the ratio of the last two terms in Eq. (1). The measured beam current is approximately proportional to the aperture size squared, while the emittance scales linearly with aperture size. Thus, and as tabulated in the last column of Table I, the effect of space charge at the aperture plane scales with the current, making the first two beams and the last one (full beam 1, aperture 1, and full beam 2 in Table I) space charge dominated at $z=0$.
As pointed out in the Introduction, beam hollowness similar to the one reported here is observed in solenoid focusing with spherical aberration (SA) [9]. However, the nonlinear character and scaling are completely different from the phenomenon reported here. Typically, when SA is appreciable, a hollow space distribution is observed near the beam waist, before and not far from the paraxial focal point. In contrast, the rings reported here occur at a distance from the solenoid that is significantly larger than the paraxial focal length. A simple calculation shows that these focal lengths are $f_{1}=8.0 \mathrm{~cm}$, and $f_{2}=10.9 \mathrm{~cm}$, 


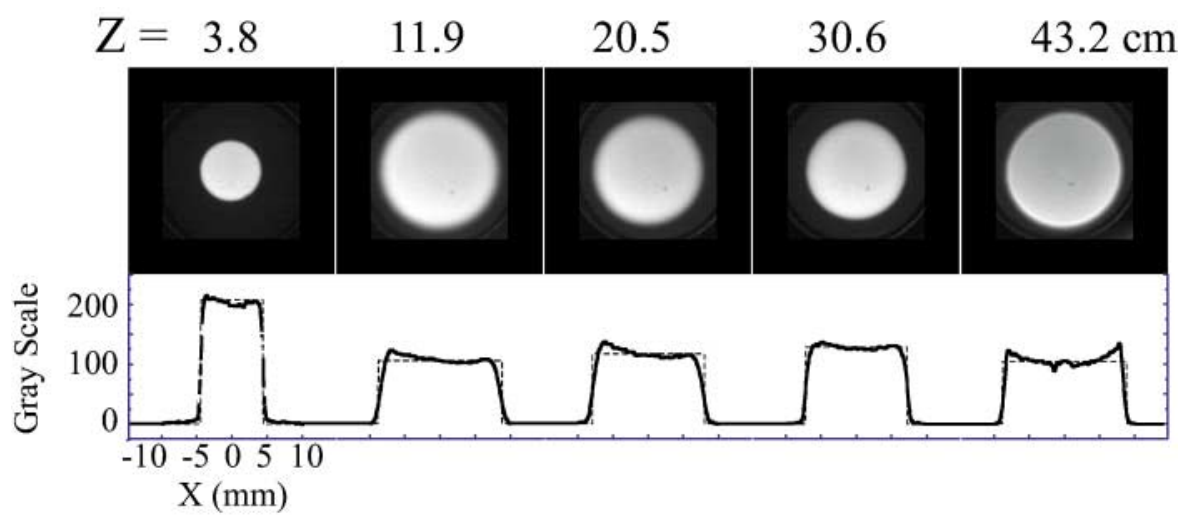

FIG. 3. (Color) Fluorescent screen pictures (top) and normalized horizontal intensity profiles (bottom) of $4 \mathrm{keV}, 17 \mathrm{~mA}$ (5 $\mu \mathrm{s}$ ) electron beam from a convergent-type gun. (See Fig. 2 for additional explanation.)

for the 5 and $4 \mathrm{keV}$ beams, respectively (the solenoid peak fields are given above). Thus, taking the plane of the apertures as the object plane $(z=0)$, the paraxial images occur at $z_{1}=24.1 \mathrm{~cm}$ and $z_{2}=23.4 \mathrm{~cm}$, instead of the observed 29 and $43.2 \mathrm{~cm}$ for the rings.
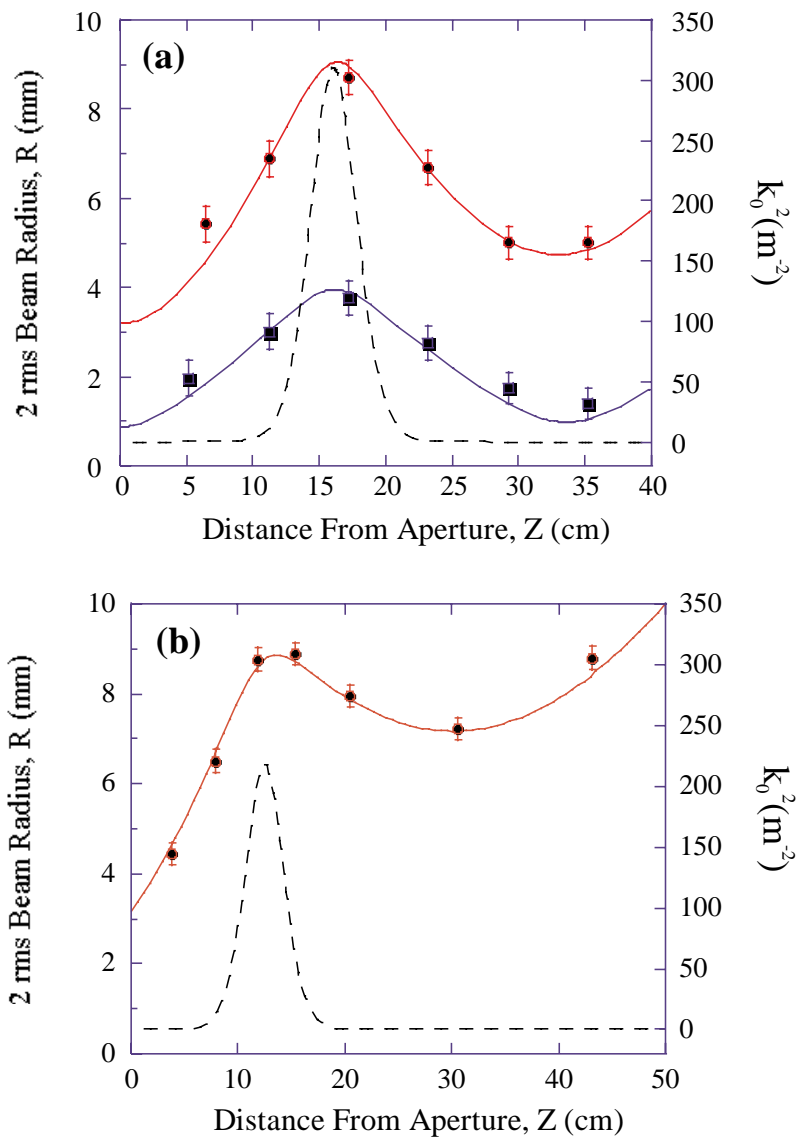

FIG. 4. (Color) KV envelope calculations and experimental ( $2 \mathrm{rms}$ ) radii for (a) full beam and smallest current beam (aperture 3 in Table I) from the gridded electron gun, and (b) full beam from the convergent-type gun. The focusing function $k_{0}^{2}(z)$ (dashed line) is proportional to the solenoid B field squared.
An initial guess of the role of the beam density profile in the formation of the ring can be extracted from Fig. 2a. From the latter, it appears as if the excess particles at the beam center eventually "diffuse" towards the edge giving rise to the hollow profile of the last pictures. However, the situation presented in Fig. 3 shows that a beam with a fairly uniform density profile can also give way to a hollow profile. Therefore, the particular shape of the initial spatial beam profile is not necessarily correlated with the appearance of hollowness: the phase space at $z=0^{+}$is required for a complete picture of the initial beam particle distribution. In other words, the current density profile must be complemented with the initial transverse velocity profile. In the absence of such detailed information, however, a model can be constructed whereby the equivalent $\mathrm{KV}$ distribution of the beam (at $z=0^{+}$) is complemented with fringe particles moving with initially steep trajectories. The tracking of these ad hoc non-KV particles reproduces correctly the appearance of rings of charge. The agreement found in every case is excellent, despite differences in electron gun configurations and some experimental conditions. Additional examples involving a focusing lattice with three solenoids and another one with six quadrupoles were reported before $[7,8]$. In what follows we describe the particle-core calculations and apply them to the solenoid experiments with the two electron guns.

The relevant differential equations for the PCM calculations in an axisymmetric (i.e., solenoidal) system are the equation for the beam envelope $R(z)$ given above [Eq. (1)] and the associated single-particle trajectory $r(z)$ (see, for example, Ref. [2]):

$$
r^{\prime \prime}(z)+k_{0}^{2} r(z)=\left\{\begin{array}{cl}
\frac{K}{R^{2}(z)} r(z), & \text { if } r(z) \leq R(z), \\
\frac{K}{r(z)}, & \text { if } r(z) \geq R(z),
\end{array}\right.
$$

where all quantities were defined before.

The results of beam edge particle tracking are shown in Figs. 5a-5e. The starting $(z=0)$ radial location, 

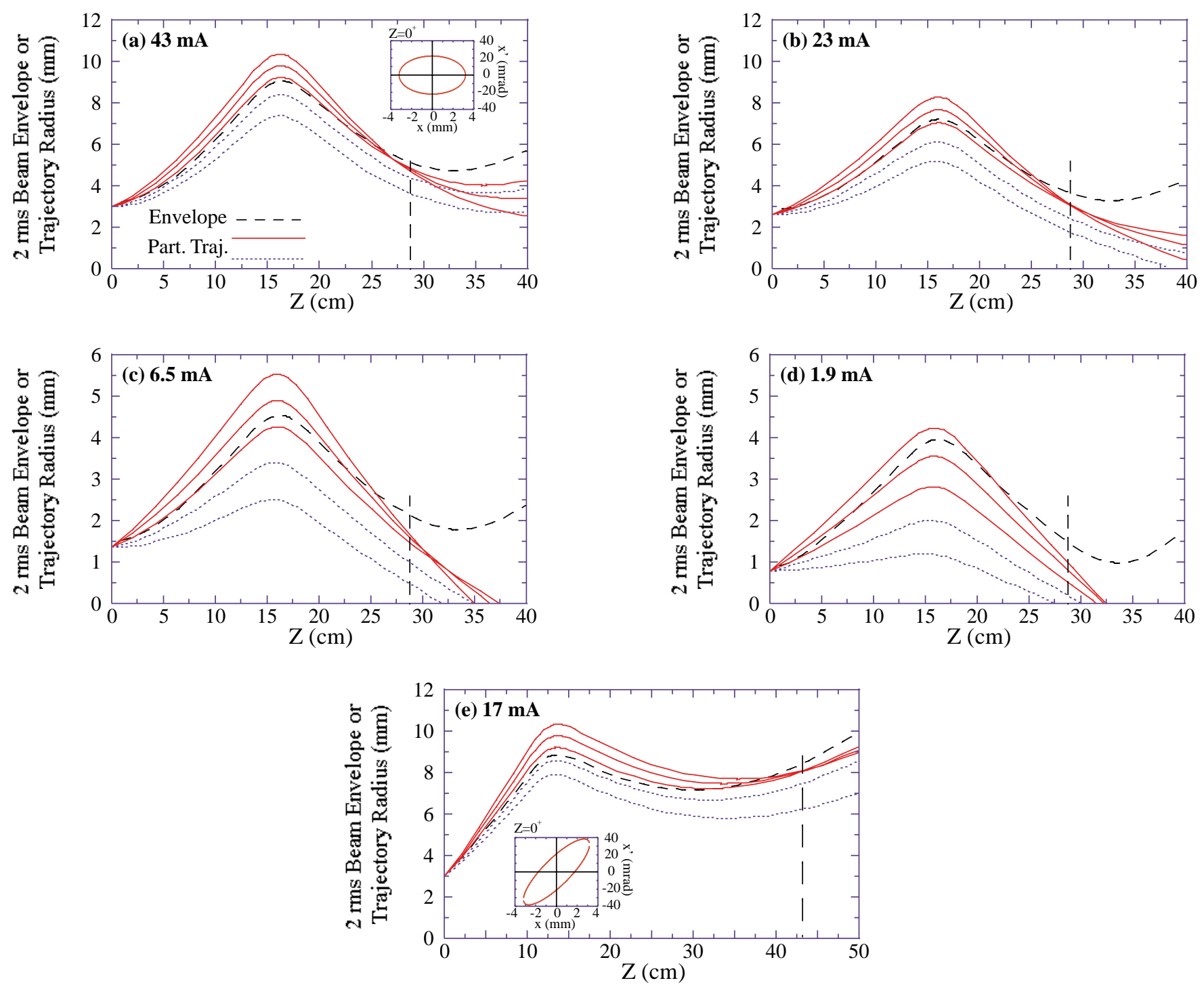

FIG. 5. (Color) Tracking of beam-edge particles [see Eqs. (1) and (2), and Table I]: (a)-(d) 5 keV electron beam from gridded gun; (e) $4 \mathrm{keV}$ electron beam from a convergent-type gun. The vertical dashed line indicates one of the planes where phosphor screen pictures were taken (see Figs. 2). In (a)-(d) the initial slopes of particle trajectories are 0, 5, 10, 15, and 20 mrad; in (e) the initial slopes are 30, 35, 40, 45, and $50 \mathrm{mrad}$. The inserts in (a) and (e) show the equivalent $\mathrm{KV}$ ellipses at $z=0^{+}$.

$r_{0}$, of the test particles inside the beam is chosen to be $0.1-0.25 \mathrm{~mm}$ from the effective beam edge. (For comparison, the Debye length is $\$ 1 \mathrm{~mm}$ in all cases, using the uniform density of the equivalent KV distribution.) This choice of $r_{0}$, together with the assumed initial values for the slopes of the trajectories, $r_{0}^{\prime}$ (see below), assures that a crossover is obtained at a location that reproduces the experimental results. The fact that the observed internal ring is not perfectly sharp but has a perceptible width could be accounted for by varying slightly the value of $r_{0}$. However, if the values of $r_{0}$, and/or $r_{0}^{\prime}$, are too small, corresponding to particles starting deep inside the beam and/or with small initial slopes, the resulting trajectories are essentially laminar, as motion occurs mostly inside the beam. This is shown in the dotted trajectories of Figs. 5a-5e.
The largest value of the initial slope used for the trajectory calculations is close to the maximum slope in the equivalent $\mathrm{KV}$ beam ellipse at the $z=0^{+}$plane (inset in Fig. 5a). This plane is close to the location of the beam waist in the experiment employing the gridded gun. Thus, the maximum value of the trajectory slope in this case is given simply by $a^{\prime}=\varepsilon / a$. From Table $\mathrm{I}$, this value is close to $a^{\prime}=20 \mathrm{mrad}$ in all cases employing the gridded gun (Figs. 5a-5d), so we choose $r_{0}^{\prime}=0,5,10,15$, and $20 \mathrm{mrad}$ as the values for the initial slopes. In the second experiment (Fig. 5e), on the other hand, the beam is expanding at the aperture plane. The envelope slope at the downstream side of this plane, or the $z=0^{+}$plane, is $32 \pm 2 \mathrm{mrad}$, as derived from measurements of the freely expanding beam. Further, the maximum slope in the equivalent KV ellipse (shown in the inset of Fig. 5e) is close 
to $40 \mathrm{mrad}$. Thus, for purposes of illustration, we go slightly above and below this value when choosing the initial slopes: $r_{0}^{\prime}=30,35,40,45$, and $50 \mathrm{mrad}$ in Fig. 5e.

From Figs. 5a and 5e, it is seen that the three edge trajectories with the steepest initial angles (solid lines) evolve completely outside the (full) beam edge, except at the sharp crossover point which occurs inside and close to the effective beam edge. As the current is reduced with the first aperture (Fig. 5b), the trajectory with initial slope equal to $10 \mathrm{mrad}$ evolves mostly inside the effective beam edge; the same applies to the case illustrated in Fig. 5c for the second aperture. For the smallest beam current (Fig. 5d), though, only the trajectory with the steepest initial angle lies outside the effective beam edge and only up to a plane close to the solenoid middle plane. Thus, no crossover is seen near the beam edge in this case. Also from Figs. 5a-5d, the value of $z$ for the crossover point is shifted downstream as the current is reduced. In other words, the focusing distance for edge imaging is shorter for larger currents. This is not easily discernible in Fig. 2, because of the limited number of pictures. However, close examination of the last two pictures in Figs. $2 \mathrm{a}$ and $2 \mathrm{~b}$ (i.e., for $z=29.2$ and $35.2 \mathrm{~cm}$ ) reveals a difference in the progression of the ring inside the beam. In Fig. 2a, the ring is blurred faster than in Fig. 2b, consistent with the way trajectories diverge after the crossover in Figs. 5a and 5b. Furthermore, additional pictures not included in Fig. 2a indicate that the sharpest ring for the $43 \mathrm{~mA}$ beam may actually occur at $z=28 \mathrm{~cm}$ instead of $z=29.2 \mathrm{~cm}$. In conclusion, edge imaging is best defined for the largest currents, for which the focusing distance is shortest.

We emphasize that the model makes no assumption whatsoever about the origin of the nonlaminar trajectories associated with the edge particles. Although we claim that space charge is a key factor that provides a lensing effect, as explained in the next section, the origin of the fringe particles is a separate matter. Further, the equivalent KV distributions include the beam-edge particles, so these particles are external to the beam only for the PCM calculations. This and the fact that the PCM constructions reproduce well the onset of the rings prove that the flow of edge particles corresponds to a small fraction of the total beam current. Thus, the lack of self-consistency of the PCM calculation is not critical.

Additional trajectories are shown in Fig. 6 for the first experiment. The solid lines represent trajectories with initial slopes equal to the maximum values consistent with the KV ellipse (inset of Fig. 5a). The dotted lines illustrate trajectories starting at $r=0$, the beam center, with slopes $r^{\prime}<22 \mathrm{mrad}$. The plot shows how the KV trajectories can yield only a uniform beam profile (families of trajectories starting at $r>0$ with initial slopes other than the maximum were omitted for the sake of clarity). Furthermore, the existence of a wide range of slopes at $r=0$, but a single value of slope at the beam edge, is simply a manifestation of the parabolic temperature profile

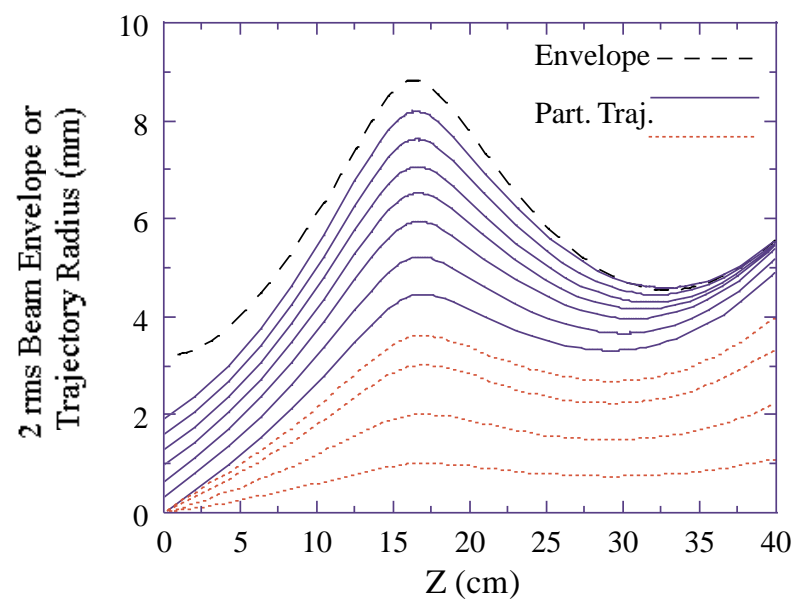

FIG. 6. (Color) KV trajectories: tracking of core particles with solenoid focusing in the gridded gun experiment. The solid lines correspond to trajectories with initial slopes equal to the maximum values consistent with the KV ellipse at $z=0^{+}$(see inset in Fig. 5a). A uniform density profile at every $z$ plane can be constructed if other trajectories (e.g., dotted lines) are included. These additional trajectories illustrate the existence of temperature in the beam.

that characterizes the KV distribution. The edge trajectories that yield the crossovers (Figs. 5a-5e) are non-KV, i.e., they are outside the KV model. The experiments and the PCM calculations presented show that real beams have nonzero temperature at the effective beam edge. In other words, the emergence of a ring of charge in space is a manifestation of a nonuniform profile in velocity.

\section{EDGE IMAGING AND THE SMOOTH APPROXIMATION}

Further insights can be gained if the ray tracing is applied to the uniform focusing case, which can be considered the smooth approximation of a periodic focusing lattice [2]. In this approximation, the constant radius of the matched beam is equal to

$$
a=\frac{1}{k_{0}}\left[\frac{K}{2}+\sqrt{\varepsilon^{2} k_{0}^{2}+\left(\frac{K}{2}\right)^{2}}\right]^{1 / 2},
$$

where all quantities were defined above. In addition, an intensity parameter can be defined [12] as

$$
\chi \equiv \frac{K}{k_{0}^{2} a^{2}}=2\left[1+\sqrt{1+\left(\frac{2 k_{0} \varepsilon}{K}\right)^{2}}\right]^{-1}
$$

which is essentially the ratio of space charge to externalfocusing forces [see Eq. (1)]. For given constant focusing $k_{0}, \chi$ increases as the ratio $K / \varepsilon$ increases. Thus, the intensity parameter can be varied with collimation, since the current scales with the aperture size squared, while the emittance scales linearly with the same quantity. 
Deviations from this scaling may occur in practice because of the nonuniform current density profiles encountered. In our case, some deviations are apparent (see Table I).

The ratio of wave numbers with and without space charge $k / k_{0}$, or tune depression, and the ratio $k_{p} / k_{0}$, where $k_{p}$ is the wave number associated with plasma oscillations, can be expressed in terms of $\chi$ :

$$
\frac{k}{k_{0}}=\sqrt{1-\chi}, \quad \frac{k_{p}}{k_{0}}=\sqrt{2 \chi} .
$$

The focal distance for edge imaging is to zero order equal to one-half the undepressed betatron wavelength, $\lambda_{0} / 2$. It becomes increasingly smaller than $\lambda_{0} / 2$ as $\chi$ is increased. This conclusion can be easily understood in terms of a linearized model for the motion of the beam edge particles. If we denote by $a$ the (constant) beam radius and move the radial coordinate origin to the beam edge, so $r=\rho+a$, we have from the second of Eqs. (2):

$$
\rho^{\prime \prime}(z)+k_{0}^{2}(\rho+a)-\frac{K}{(\rho+a)}=0,
$$

or

$$
\begin{gathered}
\rho^{\prime \prime}(z)+\left(k_{0}^{2}+\frac{K}{a^{2}}\right) \rho(z)+\left(k_{0}^{2}-\frac{K}{a^{2}}\right) a \approx 0, \\
\rho / a \ll 1 .
\end{gathered}
$$

First to be noted is that Eq. (7) is exact for $\chi=0$ and with no restriction on $\rho$. Second, the solution to the inhomogeneous Eq. (7) is straightforward:

$\rho(z)=[\rho(0)+\Gamma a] \cos \left(\beta_{E} z\right)+\frac{\rho^{\prime}(0)}{\beta_{E}} \sin \left(\beta_{E} z\right)-\Gamma a$,

where

$$
\beta_{E} \equiv k_{0}(1+\chi)^{1 / 2}, \quad \Gamma \equiv \frac{1-\chi}{1+\chi} .
$$

Therefore, from the first of Eqs. (9), the focal length for edge imaging is

$$
l_{E}=\frac{\lambda_{0}}{2}(1+\chi)^{-1 / 2} .
$$

Edge imaging should be distinguished from core imaging. From the first equality in Eq. (2), and after setting $R=a$, constant (smooth approximation), we obtain the focal distance for particle trajectories completely inside the beam:

$$
l_{C}=\frac{\lambda_{0}}{2}(1-\chi)^{-1 / 2} .
$$

Image formation is normally expected at integer multiples of this distance [13]. Notice that the factor on the righthand side is the inverse of the tune depression.

From Eqs. (10) and (11) we have

$$
\begin{aligned}
& l_{E}=\left\{\begin{array}{cc}
\lambda_{0} / 2, & \text { if } \chi=0, \\
\lambda_{0} / 2 \sqrt{2}, & \text { if } \chi=1 .
\end{array}\right. \\
& l_{C}=\left\{\begin{array}{cc}
\lambda_{0} / 2, & \text { if } \chi=0, \\
\infty, & \text { if } \chi=1 .
\end{array}\right.
\end{aligned}
$$

From Eq. (5), $l_{E}=\lambda_{P} / 2$, if $\chi=1$, i.e., in the limit of laminar motion.

In conclusion, for constant external focusing and matched beam conditions, the focusing of fringe particles increases with space-charge intensity. Physically, lensing occurs because the repulsive force of space charge decreases with radius outside the effective beam edge.

Furthermore, from Eq. (8), the radial location of the focal point for edge imaging occurs at

$$
\rho\left(l_{E}\right)=-2 a \Gamma-\rho(0) \approx-2 a \Gamma,
$$

from the beam edge. Thus, from Eqs. (12) and (9), $\rho\left(l_{E}\right) \approx-2 a$, for $\chi=0$, and $\rho\left(l_{E}\right) \approx 0$ for $\chi=1$. In the first case, fringe particles cross the beam axis and are focused near the opposite edge, while in the second case particles remain very close to the beam edge.

We consider two numerical examples corresponding to space-charge dominated and emittance-dominated beam transport. The results of PCM calculations are shown in Fig. 7.

The intensity parameter together with the constant beam radius and constant zero-current phase advance per period determines the beam dynamics in the smooth approximation. In other words, if $\chi, a$, and $k_{0}$ are given, then the perveance and emittance of the matched beam are fixed [see Eqs. (3) and (4)]. The first example has $\chi=0.7$, $a=5 \mathrm{~mm}$, and $\sigma_{0}=76^{\circ}$. These parameters correspond to $K=3.0 \times 10^{-4}$ and $\varepsilon=57 \mu \mathrm{m}$, so we choose $r_{0}^{\prime}=$ 8,10 , and $12 \mathrm{mrad}$ for the initial slopes of the edge trajectories. The PCM calculations employing Eqs. (1) and (2) and illustrated in Fig. 7a then give $l_{E}=67 \mathrm{~cm}$ or 2.1 in units of $S=0.32 \mathrm{~m}$, the full lattice period in the University of Maryland electron ring (UMER) [14]. For comparison, Eq. (10) yields $l_{E}=58 \mathrm{~cm}$ or $1.8 S$. The distance for core imaging is $l_{C}=1.38 \mathrm{~m}$ or $4.32 S$ from Eq. (11). Although no experimental evidence of edge imaging in, e.g., a long solenoid is available, particle-in-cell computer simulations in a uniform focusing channel with parameters similar to those in UMER agree well with PCM calculations $[7,8]$.

The second example has $\chi=0.10$. If the focusing strength is unchanged, the new intensity parameter is consistent with a current almost 20 times smaller than in the first example and a constant beam radius equal to $3.0 \mathrm{~mm}$ (see Fig. 7b). In this case, which corresponds to emittance-dominated transport, the focal distance becomes very close to one-half the undepressed betatron wavelength, $\lambda_{0}$, or about $76 \mathrm{~cm}$. This is expected as particle motion is now determined almost exclusively by the 

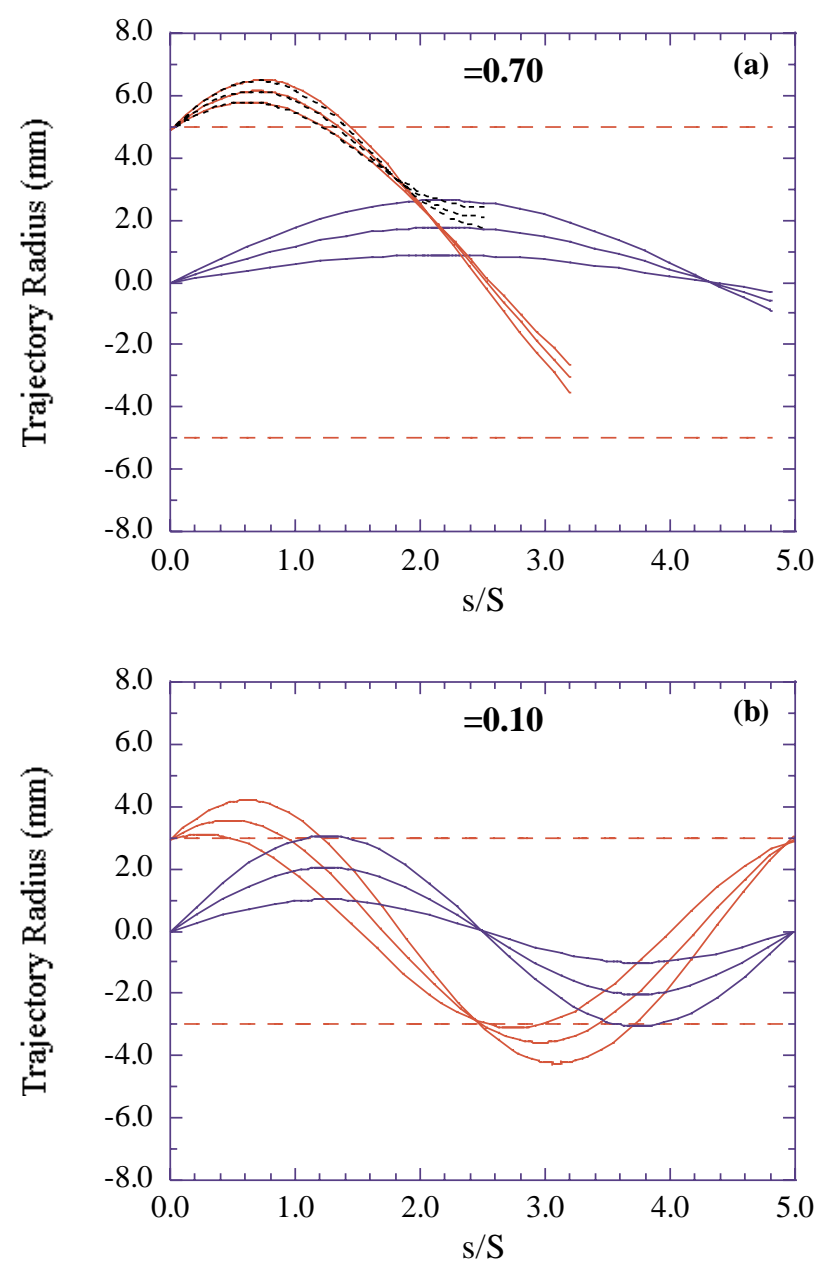

FIG. 7. (Color) Examples of edge and core imaging in a uniform focusing channel for (a) space-charge dominated transport, $\chi=$ 0.7, $a=5 \mathrm{~mm}$, and (b) emittance-dominated transport, $\chi=$ $0.1, a=3 \mathrm{~mm}$. The channel is the smooth approximation of a lattice with full lattice period $S=0.32 \mathrm{~m}$, and $\sigma_{0}=76^{\circ}$. The solid lines correspond to PCM calculations [Eqs. (1) and (2)] while the dotted curves in (a) are plots of Eq. (8). The horizontal broken lines in both (a) and (b) represent the beam edge.

external focusing. As before, however, edge imaging would depend on the existence of a significant number of fringe particles with steep trajectories at $z=0^{+}$, i.e., on the existence of a temperature profile. This is what is assumed for the calculations leading to Fig. $7 \mathrm{~b}$ where the initial slopes of the edge trajectories are $r_{0}^{\prime}=4,8$, and $12 \mathrm{mrad}$, the last value being close to $a^{\prime}=\varepsilon / a$, the maximum trajectory slope at $r=0$.

Finally, PCM calculations in the smooth approximation for values of $\chi$ around 0.5 , i.e., when the space charge and emittance terms in the rms envelope equation have comparable weights, also yield trajectory crossovers. However, the crossovers take place deep inside the beam, so the lack of self-consistency in the calculations becomes an issue. Thus, edge imaging is better defined and visualized in the regime of strongly space-charge dominated transport.

\section{CONCLUSIONS}

Although the KV distribution is a good model for the evolution of the rms envelope of any beam, it does not correctly model the actual transverse velocity profiles. In real distributions, a significant number of particles may exist around the effective ( 2 rms radius) beam edge with trajectories as steep as those at the beam core. (We emphasize that this is plausible especially near the source, where departure from equilibrium conditions is more pronounced.) Therefore, the equivalent KV distribution of a real beam can be supplemented by assuming the existence of paraxial but nonlaminar trajectories near the beam edge, starting at or near the output plane of the source. This is equivalent to introducing a nonzero temperature at the effective beam edge which, as the experiments and the PCM calculations presented show, leads to the appearance of a ring of charge in space. We call the phenomenon edge imaging as it involves linear external focusing and lensing from linearized space-charge forces outside the effective beam edge. The semiempirical model provides a visual understanding of commonly observed nonuniformities in experiments involving beams from high-perveance guns. Furthermore, the use of an ideal uniform focusing channel (or the smooth approximation of a real periodic lattice) makes possible the derivation of a simple expression for the focal length of edge imaging as a function of intensity parameter. The scaling found is contrasted with the corresponding one for the better known core imaging. Finally, the ray tracing of the model depends on the existence of space charge and, therefore, has no counterpart in light optics. Questions remain on the origin of the fringe particles, i.e., on the emergence of nonuniform temperature profiles in real beams, on the implications for the long term evolution of the beam, and on possible uses of the phenomenon for beam diagnostics.

\section{ACKNOWLEDGMENTS}

We wish to acknowledge helpful assistance in the experiments and mechanical design from M. Virgo and V. Yun. We also thank A. Valfells, R. Kishek, and I. Haber for useful discussions. The work is supported by the U.S. Department of Energy.

[1] See, for example, Hermann Wollnik, Optics of Charged Particles (Academic Press, New York, 1987), and references therein.

[2] M. Reiser, Theory and Design of Charged-Particle Beams (Wiley, New York, 1994), and references therein.

[3] I. M. Kapchinskij and V. V. Vladimirskij, in Proceedings of the International Conference on High Energy Accelerators, Geneva, 1959 (CERN, Geneva, 1959), p. 274.

[4] Frank J. Sacherer, IEEE Trans. Nucl. Sci. 18, 1105 (1971).

[5] J.S. O'Connell et al., in Proceedings of the 1993 Particle Accelerator Conference, Washington, D.C., 1993 (IEEE, New York, 1993), p. 365. 
[6] T.P. Wangler, K. R. Crandall, R. Ryne, and T.S. Wang, Phys. Rev. ST Accel. Beams 1, 084201 (1998).

[7] S. Bernal, R. A. Kishek, M. Reiser, and I. Haber, Phys. Rev. Lett. 82, 4002 (1999).

[8] S. Bernal, P. G. O'Shea, R. Kishek, and M. Reiser, in Advanced Accelerator Concepts: Ninth Workshop Santa $\mathrm{Fe}$, NM, 2000, AIP Conf. Proc. No. 569 (AIP, New York, 2001), p. 544.

[9] P. Loschialpo, W. Namkung, M. Reiser, and J. D. Lawson, J. Appl. Phys. 57, 10 (1985).

[10] O. A. Anderson, Part. Accel. 21, 197 (1987).
[11] S. Bernal, P. Chin, R. A. Kishek, Y. Li, M. Reiser, J. G. Wang, T. Godlove, and I. Haber, Phys. Rev. ST Accel. Beams 1, 044202 (1998).

[12] M. Reiser et al., in Proceedings of the 1999 Particle Accelerator Conference, New York, 2000 (IEEE, New York, 2000), p. 234.

[13] I. Haber, D. Kehne, M. Reiser, and H. Rudd, Phys. Rev. A 44, 5194 (1991).

[14] P. G. O'Shea et al., Nucl. Instrum. Phys. Res. A 464, 646-652 (2001). 